\title{
Massive Stellar Clusters and Superwind Engines in the Antennae
}

\author{
Andrea M. Gilbert \\ Max-Planck Institut für extraterrestrische Physik, Garching, Germany
}

James R. Graham

University of California, Berkeley, USA

\begin{abstract}
The youngest super star clusters (SSCs) in the Antennae Galaxies (NGC 4038/39) drive supersonic mass-loaded outflows. Highresolution Keck/NIRSPEC spectroscopy reveals broad, spatially extended $\mathrm{Br} \gamma$ emission lines that are well-fit by simple wind models. Cluster massloss rates are up to $1.5 \mathrm{M}_{\odot} \mathrm{yr}^{-1}$ and terminal velocities are up to 205 $\mathrm{km} \mathrm{s}^{-1}$. These emission-line clusters (ELCs) constitute at least $15 \%$ of the star formation rate in the Antennae, and their high star formation efficiencies imply that they will probably evolve into bound SSCs. The youngest ELC outflows are driven primarily by stellar winds, and they efficiently entrain ambient matter. They transfer or dissipate most of their energy and momentum in a hot or cool medium that does not emit $\operatorname{Br} \gamma$. ELCs are the individual engines that power galactic-scale superwinds, viewed in their earliest evolutionary stage.
\end{abstract}

\section{Mass-Loaded Outflows from Emission-Line Clusters}

We discovered broad, supersonic Br $\gamma$ emission in Keck/NIRSPEC echelle spectra of the youngest SSCs in the Antennae (Gilbert 2002). The $\mathrm{Br} \gamma$ profiles are well resolved (spectral resolution was $12 \mathrm{~km} \mathrm{~s}^{-1}$ ), have widths of $60-105 \mathrm{~km} \mathrm{~s}^{-1}$ FWHM, and exhibit high-velocity non-Gaussian wings (Fig. 1). We dub these broad-line SSCs emission-line clusters (ELCs). Our incomplete sample of ELCs comprises $15 \%$ of the total SFR in the Antennae, and estimates of their starformation efficiency (30-70\%) suggest that they may evolve into bound SSCs, which in turn may evolve into globulars (Gilbert 2002). ELCs are typically more compact $(d \sim 40-80 \mathrm{pc})$, denser, and brighter than giant extragalactic $\mathrm{H}$ II regions (GHIIRs), with $\mathrm{Q}=10^{51}-10^{53} \mathrm{~s}^{-1}$, and their recombination line fluxes and widths exhibit the same correlation seen for GHIIRs (Gilbert 2002).

$\mathrm{Br} \gamma$ line fluxes and $\mathrm{K}$ magnitudes imply ELC ages of 3-8 Myr and masses of $10^{5}-10^{7} \mathrm{M}_{\odot}$ (using Starburst99 models, Leitherer et al. 1999). Comparing the virial line widths (from ELC masses and sizes) with observed ones suggests that much of the gas in ELC outflows is unbound.

We determine the mass-loss rates of ELCs, by modeling their supersonic $\mathrm{Br} \gamma$ profiles with a semi-empirical $\beta$-wind law that is successful for radiation-driven wind lines of hot stars (e.g. Kudritzki \& Puls 2000), and provides excellent fits to 

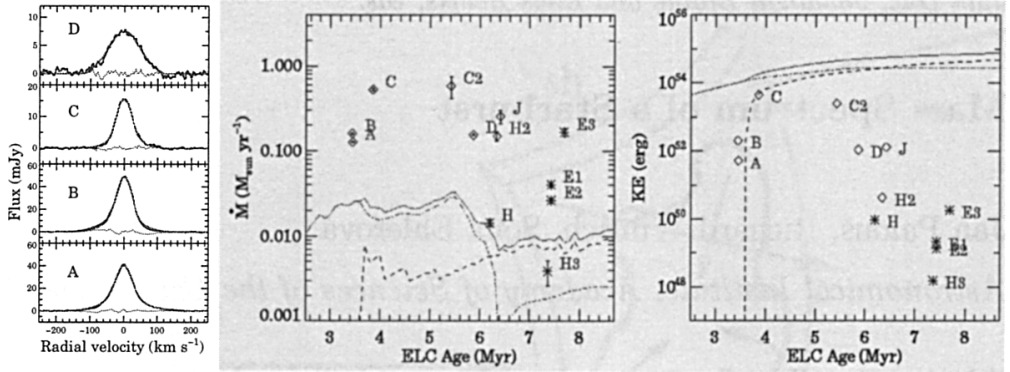

Figure 1. Supersonic $\operatorname{Br} \gamma$ profiles of four ELCs with overplotted wind models and fit residuals (left). Mass-loss rates (center) and kinetic energy (right) measured for ELC outflows (points, normalized to a $10^{6}$ $\mathrm{M}_{\odot}$ cluster). Curves are predictions from Starburst99 for a cluster's stellar winds (dotted), SNe (dashed), and their sum (solid).

our spectra (Fig. 1). ELC mass-loss rates in ionized gas $\left(\dot{M}_{\mathrm{HII}}\right)$ are $0.006-1.5$ $\mathrm{M}_{\odot} \mathrm{yr}^{-1}$. Fig. 1 compares observed normalized $\dot{M}_{\mathrm{HII}}$ with that provided by stellar ejecta (winds and supernovae) in a Starburst99 cluster of $10^{6} \mathrm{M}_{\odot}$. For most ELCs we detect over an order of magnitude more outflowing photoionized gas than is ejected directly from stars; thus ELC outflows efficiently entrain matter. Either older ELCs entrain matter less efficiently, or they have swept away most of their reservoir of neutral gas. Another indication that we are not observing mass lost directly from hot stars comes from the low $\left(\leq 205 \mathrm{~km} \mathrm{~s}^{-1}\right)$ terminal velocities of ELC outflows. They are about ten times less than the terminal velocity of a single $\mathrm{O}$ star wind, and is probably due to the interaction of many individual winds, photoevaporative flows, (in older clusters) supernova explosions, and ultimately the collision with ambient neutral gas.

Fig. 1 also displays the measured and predicted kinetic energies (KEs) of the outflows. In $\mathrm{Br} \gamma$ we detect only a small fraction of the $\mathrm{KE}$ available from winds and SNe: ELC outflows have a high thermalization efficiency. The rest of the KE must reside in a hotter and/or cooler medium. Stellar wind shocks generate X-rays, and Chandra observations reveal possible counterparts for some ELCs (e.g. Zezas et al. 2002). Efficient thermalization of mechanical energy in mass-loaded winds is common in large-scale superwinds (e.g. M82, Suchkov et al. 1996), the earliest phase of which may be taking place in the Antennae.

Acknowledgments. This work was supported in part by a NASA GSRP Fellowship and NSF grants AST-0205999 and AST-9876783.

\section{References}

Gilbert, A. M. 2002, Ph.D. Thesis (University of California, Berkeley)

Kudritzki, R. \& Puls, J. 2000, ARA\&A, 38, 613

Leitherer, C., et al. 1999, ApJS, 123, 3

Suchov, A. A., et al. 1996, ApJ, 463, 528

Zezas, A., et al. 2002, ApJ, 577, 710 